\title{
Aberration-Corrected STEM Imaging and Spectroscopy of Single-Layered Materials
}

\author{
M.F. Chisholm, ${ }^{*}$ G. Duscher, ${ }^{* *}$ and W. Windl***
}

* Materials Science and Technology Division, Oak Ridge National Laboratory, PO Box 2008, Oak Ridge, TN 37831-6069

** Department of Materials Science and Engineering, University of Tennessee, Knoxville, TN 37996-2200

*** Department of Materials Science and Engineering, The Ohio State University, Columbus, $\mathrm{OH}$ 43210

Aberration-corrected scanning transmission electron microscopes can now produce electron probes as small as $1 \AA$ at $60 \mathrm{keV}$. This level of performance has been shown to allow individual atoms to be imaged in various novel materials.[1] Examples of graphene, monolayer boron nitride and carbon nanotubes will be used to show that, in this microscope optimized for low-voltage operation, the ADF signal is strong enough to image and identify the chemical type of all atoms present in the layer. Fig. 1a is a medium angle annular dark field image recorded at $60 \mathrm{kV}$. This acceleration voltage is below the knock-on damage threshold for these single-layer materials. Individual $\mathrm{C}$ atoms are clearly seen - as is the lone impurity atom that has substituted for carbon. Using an earlier derived intensity model, this impurity is identified as a silicon atom. This identification is confirmed by EELS.

While an occasional impurity can be found to replace carbon in an otherwise perfect graphene layer, most impurities are found at structural defects in the layer. For example Fig. 1b shows a symmetric cluster of four dislocations in graphene. These 5- and 7-atom rings in various combinations are the most numerous defects seen in graphene. Interestingly, no Stone-Wales defects [2] (a specific pairing of two 5/7 atom rings predicted to be a common defect in graphene and single-walled nanotubes) were seen. In Fig. 1c three types of impurity atoms are detected incorporated in the graphene layer: six $\mathrm{Si}$ atoms [apparently a common impurity in graphene], three $\mathrm{N}$ atoms and a single $\mathrm{S}$ atom.

And of course graphene rolled into a tube or a horn can be studied in the same way. Fig. 1d is an image of a single-walled carbon nanohorn. The image is a superposition of the front and back surfaces of the structure.[3] The Fourier transform of the nanotube can be used to dertermine the chirality of the nanohorn. This structure is seen to have a single bright atom incorporated in the carbon lattice. The image and EELS spectrum can be analyzed and is seen to be consistent with having a single $\mathrm{Si}$ atom in this specific region.

We have shown that it is possible to distinguish individual $\mathrm{B}$ and $\mathrm{N}$ atoms in monolayer $\mathrm{BN}$ and detect $\mathrm{C}$ and $\mathrm{O}$ impurities in the monolayer.[1] This effort has been extended showing that a range of atoms from $\mathrm{B}$ to $\mathrm{Cu}$ in and on graphene can also be identified from the image alone. Within this range of elements of $\mathrm{B}$ to $\mathrm{Cu}$, the intensity of individual atoms was seen to vary as the atomic number of the element, $Z$, to the power of $\sim 1.64$.[4] 
References

[1] O.L. Krivanek et al., Nature. 464 (2010) 571.

[2] A.J. Stone and D.J. Wales, Chemical Physics Letters. 128 (5-6) (1986) 501.

[3] A. Hashimoto et al., Nature, 430 (2004) 871.

[4] This research was supported by the Materials Science and Technology Division of the U.S. Dept. of Energy and in part by NSF Award number DMR-0925529 and the Center for Emergent Materials at The Ohio State University, an NSF MRSEC.

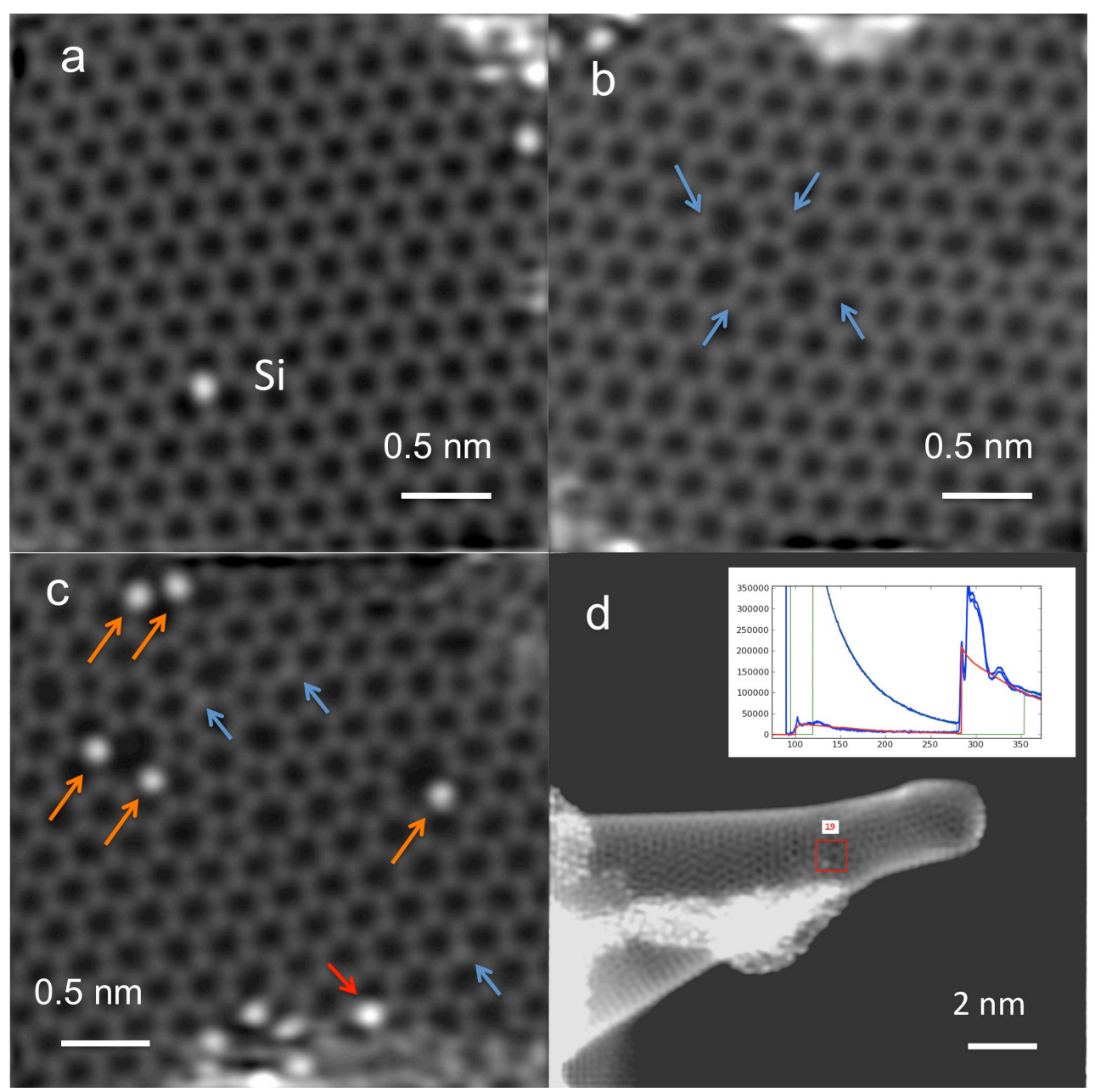

FIG. 1. ADF images of graphene processed to remove noise and probe tail effects. (a) Single Si substitutional defect. (b) Symmetric collection of four 5/7 atom ring defects. (c) Three impurity types located at structural defects. There are 5 arrowed $\mathrm{Si}$ atoms, $3 \mathrm{~N}$ atoms and a $\mathrm{S}$ atom. (d) image of a single-wall carbon nanohorn. The indicated region in red produced the EELS spectra seen in the insert. The analyzed Si/C ratio is 0.6 atom \% . Thus, with 144 atoms in analyzed region, the EELS data indicates that there is one $\mathrm{Si}$ atom. 